\title{
El ataque posicional en balonmano: validación de un sistema de observación
}

\section{The positional attack in handball: validation of an observation system}

\section{O ataque posicional no andebol: validação de um sistema de observação}

\author{
Quiñones, Y. ${ }^{1}$, Morillo-Baro, J. P. ${ }^{2}$, Reigal, R. E. ${ }^{3}$, Morales-Sánchez, V. ${ }^{4}$, Vázquez-Diz, J. A. , \\ Hernández-Mendo, A. ${ }^{6}$ \\ Facultad de Psicología, Universidad de Málaga ${ }^{1,2,3,4,5,6}$
}

\begin{abstract}
RESUMEN
En este estudio se presenta un instrumento de observación para analizar las acciones de ataque posicional en el balonmano de alto nivel. La herramienta ha sido diseñada ad hoc mediante un sistema mixto de formatos de campo y sistema de categorías exhaustivas y mutuamente excluyentes (E/ME). La conforman 12 criterios y 62 categorías, que proponen como principales criterios la situación que produce el desequilibrio, los medios tácticos colectivos empleados y la ejecución de la decisión. Se realizó un análisis de Calidad del Dato y un análisis de Generalizabilidad. Se estimaron los coeficientes de correlación (Pearson, Spearman, Tau b de Kendall) y el índice de Kappa de Cohen para ambos sexos. Las correlaciones estimadas para sexo masculino fueron de (.97-.99) para la fiabilidad intraobservador y (.89-.98) en la interobservador. El índice de la Kappa de Cohen resultó 0.94 y 0.82 respectivamente. El sexo femenino obtuvo en el coeficiente de correlación intraobservador (.95-.97) y (.87-.98) en el inter. El índice de la Kappa de Cohen fue 0.91 en la concordancia inter y 0,81 en la intra. Los resultados han mostrado índices adecuados de correlación, así como excelentes resultados de la Generalizabilidad con un valor G relativo y $\mathrm{G}$ absoluto de .99 en el acuerdo interobservador y 1.00 para el acuerdo intraobservador. Como aspecto novedoso, se realizó un análisis de invarianza para demostrar que no existen diferencias por razón de género en la utilización de la herramienta. Los datos generados del estudio aseguran que la herramienta de observación presenta una elevada validez, precisión y fiabilidad.
\end{abstract}

Palabras clave: Metodología Observacional, Análisis de Generalizabilidad, Análisis de Invarianza, Calidad del Dato, Balonmano.

\section{ABSTRACT}

In this study an observation instrument is presented to analyze the positional attack actions in high level handball. The tool has been designed ad hoc using a mixed system of field formats and a system of exhaustive and mutually exclusive categories (E/ME). It is made up of 12 criteria and 62 categories, which propose as main criteria the situation produced by the imbalance, the collective tactical means employed and the execution of the decision. An analysis of Data Quality and an analysis of Generalizability. Correlation coefficients (Pearson, Spearman, Tau b de Kendall) and Cohen Kappa index were estimated for both sexes. The correlations estimated for male sex were (.97$.99)$ for intra-observer reliability and (.89-.98) for interobserver reliability. Cohen's Kappa index was .94 and 0.82 respectively. The female sex obtained an intra-observer correlation coefficient of (.95-.97) and (.87-.98) in the inter. Cohen's Kappa index was 0.91 in the inter concordance and 0.81 in the intra. The results have shown adequate correlation indices as well as excellent Generalizability results with a relative $G$ and absolute $G$ value of 99 in the 


\section{Quiñones, Morillo-Baro, Reigal, Morales-Sánchez, Vázquez-Diz, Hernández-Mendo.}

inter-observer agreement and 1.00 for the intra-observer agreement. As a novelty, an invariance analysis was carried out to demonstrate that there are no gender differences in the use of the tool. The data generated from the study ensures that the observation tool is highly valid, accurate and reliable.

Keywords: Observational Methodology, Generalizability Analysis, Invariance Analysis, Data Quality, Handball.

\section{RESUMO}

Neste estudo é apresentado um instrumento de observação para analisar as ações de ataque posicional no handebol de alto nível. A ferramenta foi concebida ad hoc utilizando um sistema misto de formatos de campo e um sistema de categorias exaustivas e mutuamente exclusivas (E/ME). É composto por 12 critérios e 62 categorias, que propõem como critérios principais a situação produzida pelo desequilíbrio, os meios táticos coletivos empregados e a execução da decisão. Foi realizada uma análise da Qualidade dos Dados e uma análise da Generalizabilidade. Os coeficientes de correlação (Pearson, Spearman, Tau b de Kendall) e o índice Cohen Kappa foram estimados para ambos os sexos. As correlações estimadas para sexo masculino foram (.97-.99) para confiabilidade intra-observador e (.89-.98) para confiabilidade interobservador. O índice Kappa de Cohen foi de 0.94 e 0.82 , respectivamente. O sexo feminino obteve um coeficiente de correlação intra-observador de (.95-.97) e (.87-.98) no intervalo. O índice Kappa de Cohen foi de 0.91 na inter concordância e de 0.81 no intra. Os resultados mostraram índices de correlação adequados, bem como excelentes resultados de Generalizabilidade com um valor $G$ relativo e $G$ absoluto de 0.99 na concordância inter-observador e 1.00 para a concordância intra-observador. Como novidade, foi realizada uma análise de invariância para demonstrar que não há diferenças de gênero no uso da ferramenta. Os dados gerados a partir do estudo garantem que a ferramenta de observação é altamente válida, precisa e confiável.

Palavras chave: Metodologia Observacional, Análise de Generalizabilidade, Análise de Invariância, Qualidade dos Dados, Andebol.

\section{INTRODUCCIÓN}

En los deportes de cooperación-oposición, la evaluación del rendimiento en competición es un área de investigación que ha evolucionado en los últimos años (Casal, Maneiro, Ardá, Losada y Rial, 2014; Prieto, Gómez y Sampaio, 2015a, 2015b; Sarmento et al., 2014; Sá, Rui, Saavedra y Fernández, 2015; Tassi, Rivera y Morilla, 2018; Teles y Volossovitch, 2015; Vukosavljević, Kocić, Beric y Stojić, 2015). En estos estudios, el uso de nuevas técnicas e instrumentos de investigación han permitido la obtención de un mayor número de información necesaria para valorar la preparación de los jugadores; permitiendo redireccionar el entrenamiento de los equipos y elevar las exigencias físicas, técnico-tácticas y psicológicas (Vázquez-Diz, Morillo-Baro, Reigal, MoralesSánchez y Hernández-Mendo, 2019b, 2019c).

De manera significativa se realizan estudios descriptivos y comparativos mediante la Metodología Observacional (MO), que permiten el análisis de los resultados de los campeonatos de alto nivel (Anguera y Hernández-Mendo, 2013; Anguera y HernándezMendo, 2015). La MO es un procedimiento científico, flexible y riguroso en el estudio del juego (Anguera y Hernández-Mendo, 2014; Anguera, BlancoVillaseñor, Hernández-Mendo y Losada 2011) que posibilita analizar los comportamientos que suceden en el deporte en su contexto natural mediante la creación de herramientas de observación ad hoc (Anguera y Hernández-Mendo, 2013).

La utilización de la MO en el estudio de la actividad física y los deportes es un recurso cada vez más demandado. Los deportes de equipo se han valido de ella para analizar los factores determinantes en el rendimiento a través del diseño y validación de sistemas de observación (Ortega-Toro, GarcíaAngulo, Gimenez-Egido, García-Angulo y Palao, 2019). En el trabajo de Casal, Maneiro, Ardá, Losada y Rial (2014) se propone identificar las variables asociadas al resultado en los tiros libres indirectos en el fútbol de élite. En el estudio de (Moreno y Gómez, 2017; Muñoz y Serna, 2015) han analizado los lanzamiento a partir de las variables asociadas al desarrollo del juego en el baloncesto profesional. En voleibol, Pajares, Echeverría, Silva, Suárez y Arroyo (2017) estudian las variables relativas a la recepción que actúan como predictivas. La investigación de Santos, Sarmento, Alves y Campaniço (2014) se centra en el estudio de la dinámica del desempeño individual y colectivo en waterpolo, y en el trabajo de Villarejo, Ortega, Gómez y Palao (2014) se analiza las posesiones del balón en rugby.

En balonmano se han analizado los comportamientos deportivos en contextos naturales, mostrando 


\section{El ataque posicional en balonmano: validación de un sistema de observación}

excelentes resultados. La revisión de la literatura científica específica de este deporte muestra las propuestas de Marques (2011) para analizar las secuencias de la defensa de la selección española en los Juegos Olímpicos de Beijing; para analizar la eficacia del transporte del balón durante el contrataque (González, 2012); la importancia de las finalizaciones de los jugadores extremos en la fase de ataque (Montoya, Moras y Anguera, 2013); la herramienta de observación de Jiménez-Salas y Hernández-Mendo (2016) para realizar registros fiables de las acciones ofensivas del contraataque; y la identificación de las diferencias entre mujeres $y$ hombres en las competiciones de balonmano de alto nivel (Pic, 2017). En la modalidad de balonmano playa, Morillo y Hernández-Mendo (2015) crean un sistema de observación para realizar registros fiables de las acciones ofensivas y Vázquez-Diz, Morillo-Baro, Reigal, Morales-Sánchez, y Hernández-Mendo (2019a) direccionan el instrumento de observación a las acciones del portero.

Las investigaciones que se realizan en balonmano muestran un mayor número de estudios del juego ofensivo en comparación con la fase defensiva (Prieto, Gómez y Sampaio, 2015); una de las razones es por presentar en su escenario las acciones más complejas y lúcidas del juego. De igual modo, se estudian los comportamientos técnico-tácticos ofensivos de los jugadores desde una perspectiva ecológica, aportando informaciones de gran utilidad para el estudio de este deporte: las variables que intervienen en la eficacia de los sistemas ofensivos estructurado, no estructurado y contraataque (Lozano y Camerino, 2012); el comportamiento táctico ofensivo en alto rendimiento y su relación con los diferentes factores de rendimiento (Lozano, 2014; Lozano, Camerino e Hileno, 2016); el desempeño individual técnicotáctico (Tulha Duarte Ferreira, Braga Santos Graça y Dias Estriga, 2018); la eficacia del ataque y los lanzamientos (González, Botejara, Martínez y Chirosa, 2016); y la eficacia del ataque en competiciones panamericanas (González y Ramírez, 2016).

Los aportes de estas investigaciones permiten continuar el estudio de las acciones técnico-tácticas durante el ataque posicional, donde se construye el juego colectivo organizado desde los puestos específicos. Sus resultados han mostrado el interés de observar las diversas situaciones de finalización de los jugadores, siendo el lanzamiento una categoría con grandes índices de ocurrencia. Específicamente, en esta investigación se propone el estudio de la calidad de la ejecución técnico-táctica, al mismo tiempo que se propone registrar las condiciones o situaciones en que se efectúan los lanzamientos.

Teniendo en cuenta lo anteriormente expuesto, se plantea como objetivos de la investigación: (1) Diseñar una herramienta de observación que permita registrar las acciones de los jugadores durante el ataque posicional en balonmano; cumpliendo los criterios de fiabilidad, validez y precisión; (2) Realizar el análisis de Generalizabilidad para determinar la fiabilidad de los observadores, valorar la bondad de las categorías y estimar el número de sesiones mínimas para generalizar con precisión (Blanco-Villaseñor, Castellano, Hernández-Mendo, Sánchez-López y Usabiaga, 2014).

\section{MATERIAL Y MÉTODOS}

\section{Diseño}

En el proceso de construcción de la herramienta de observación se utilizó una estrategia "empíricoinductiva". El diseño observacional (Anguera, Blanco-Villaseñor, Hernández-Mendo y Losada, 2011) que se plantea es nomotético, puntual y multidimensional.

\section{Participantes}

El análisis de la Calidad del Dato se realizó con equipos profesionales masculinos y femeninos. Se han utilizado 840 registros del equipo Ángel Ximénez AVIA Puente Genil frente al equipo Balonmano Benidorm, disputado durante la Liga de Balonmano Española ASOBAL 2018/2019. En categoría femenina se observó el partido de los equipos Rincón Fertilidad Málaga y Balonmano Castellón en la Copa S.M. La Reina 2019 en el partido de vuelta, utilizándose 802 registros.

\section{Instrumentos}

Los partidos fueron grabados por el club local y posteriormente fueron solicitados y proporcionados para su visionado y análisis. En este caso, por Ángel Ximénez AVIA Puente Genil (club local masculino) y por Rincón Fertilidad Málaga (club local femenino). Se utilizó el software informático HOISAN (Hernández-Mendo, López-López, Castellano, Morales-Sánchez y Pastrana, 2012) para el registro y 


\section{Quiñones, Morillo-Baro, Reigal, Morales-Sánchez, Vázquez-Diz, Hernández-Mendo.}

codificación de las observaciones, así como para la obtención de las correlaciones necesarias para el análisis de Calidad del Dato. Fue utilizado, además, el programa informático SAGT v1.0 (HernándezMendo, Ramos-Pérez y Pastrana, 2012) para la aplicación de la Teoría de Generalizabilidad estimando la concordancia intraobservador e interobservador, la homogeneidad de las categorías y el número mínimo de sesiones para generalizar con precisión.

Se creó una herramienta de observación ad hoc mediante un sistema mixto de formatos de campo y sistema de categorías exhaustivas y mutuamente excluyentes (E/ME) (Anguera, 1979; HernándezMendo, 1996; Castellano, 2000). Está formada por 12 criterios y 62 categorías que se corresponden con el orden secuencial desde que el balón es puesto en juego hasta que finaliza la acción. En la tabla 1 se presenta la herramienta de observación con los criterios, categorías y sistema de codificación que la conforman.

Tabla 1. Criterios, categorías y sistema de codificación de la herramienta observacional

\begin{tabular}{|c|c|c|c|}
\hline & Criterios & Códigos & Categorías \\
\hline \multirow{6}{*}{1.} & \multirow{3}{*}{ Localización } & LOC & Local \\
\hline & & VISIT & Visitante \\
\hline & & NEUTR & Neutral \\
\hline & \multirow{8}{*}{ Marcador } & EMPTE & Empate \\
\hline & & Gx1 & Ganando por un gol. \\
\hline & & $\mathrm{Gx} 2$ & Ganando por dos goles \\
\hline \multirow[t]{7}{*}{2.} & & GxM2 & Ganando por más de dos goles \\
\hline & & Px1 & Perdiendo por un gol \\
\hline & & Px2 & Perdiendo por dos goles \\
\hline & & PxM2 & Perdiendo por más de dos goles \\
\hline & & T010 & $\begin{array}{l}\text { Tiempo del partido del minuto } 0 \\
\text { al } 10\end{array}$ \\
\hline & \multirow{5}{*}{$\begin{array}{l}\text { Minuto de } \\
\text { juego }\end{array}$} & $\mathrm{T} 1120$ & $\begin{array}{l}\text { Tiempo del partido del minuto } \\
11 \text { al minuto } 20\end{array}$ \\
\hline & & $\mathrm{T} 2130$ & $\begin{array}{l}\text { Tiempo del partido del minuto } \\
21 \text { al } 30\end{array}$ \\
\hline \multirow[t]{4}{*}{3.} & & T3140 & $\begin{array}{l}\text { Tiempo del partido del minuto } \\
31 \text { al } 40\end{array}$ \\
\hline & & T4150 & $\begin{array}{l}\text { Tiempo del partido del minuto } \\
41 \text { al } 50\end{array}$ \\
\hline & & T5160 & $\begin{array}{l}\text { Tiempo del partido del minuto } \\
51 \text { al } 60\end{array}$ \\
\hline & \multirow{5}{*}{$\begin{array}{l}\text { Zona inicial } \\
\text { de la acción }\end{array}$} & TP & $\begin{array}{l}\text { El partido se encuentra en } \\
\text { tiempo de prórroga }\end{array}$ \\
\hline \multirow{4}{*}{4.} & & ZI1 & Zona inicial 1 \\
\hline & & ZI2 & Zona inicial 2 \\
\hline & & ZI3 & Zona inicial 3 \\
\hline & & DLI & Desequilibra el lateral izquierdo \\
\hline
\end{tabular}

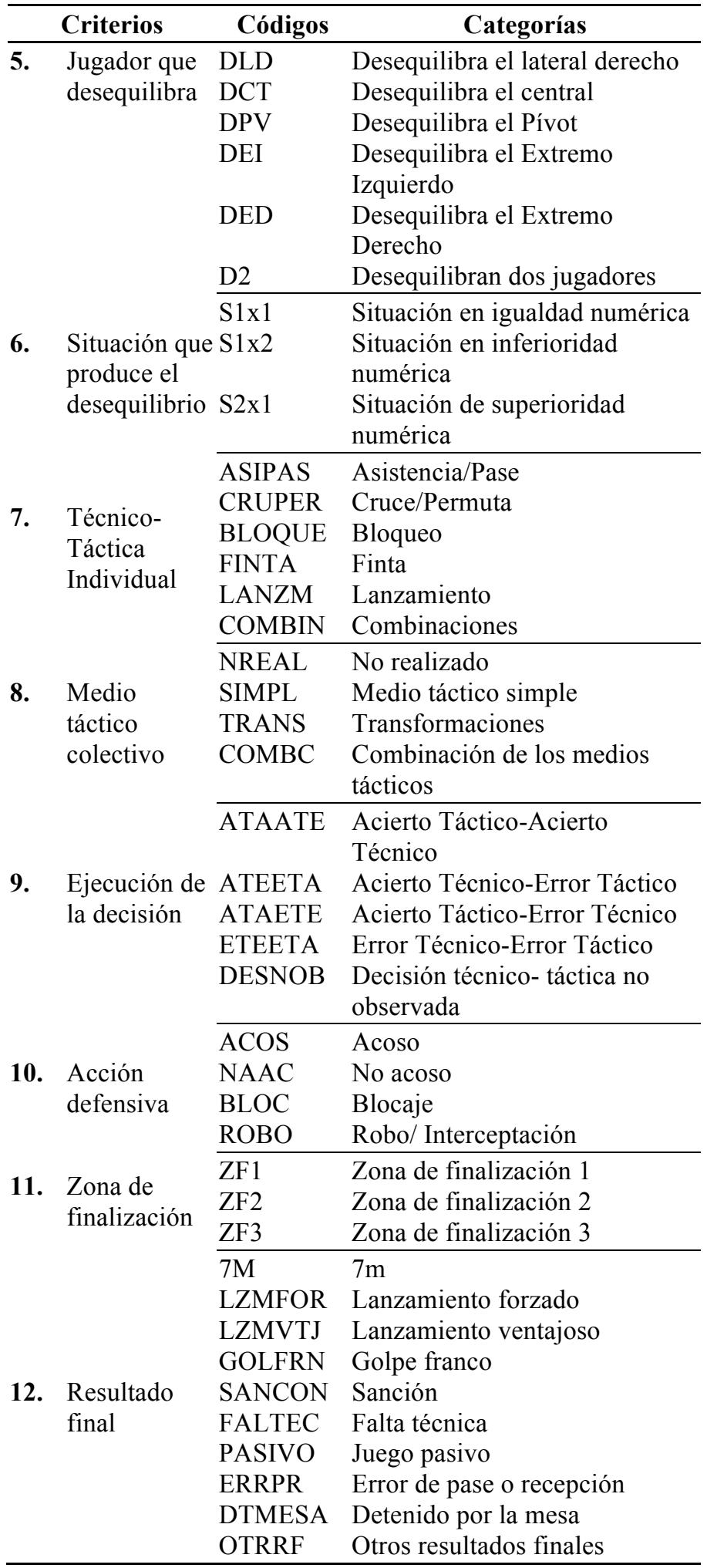

El primer criterio hace referencia a la localización; escenario donde se desarrolla el partido. El marcador es el criterio que se corresponde a la suma numérica 


\section{El ataque posicional en balonmano: validación de un sistema de observación}

de goles de cada equipo, la categoría empate (EMPTE) se propone para el marcador igualado en goles y en el inicio del partido. El minuto de juego corresponde a la franjas de tiempo del partido en que se produce el ataque, y se presentan en siete categorías que van de diez en diez minutos hasta el minuto 60 del partido, además se decidió incorporar la categoría tiempo prórroga (TP). El criterio zona inicial de la acción se refiere a las zonas donde se producen las acciones de desequilibrio. (ZI1) es el área izquierda del terreno del equipo contrario, (ZI2) es la zona central del terreno determinada por los tres metros del ancho de la portería, y (ZI3) es la zona derecha del terreno del equipo contrario.

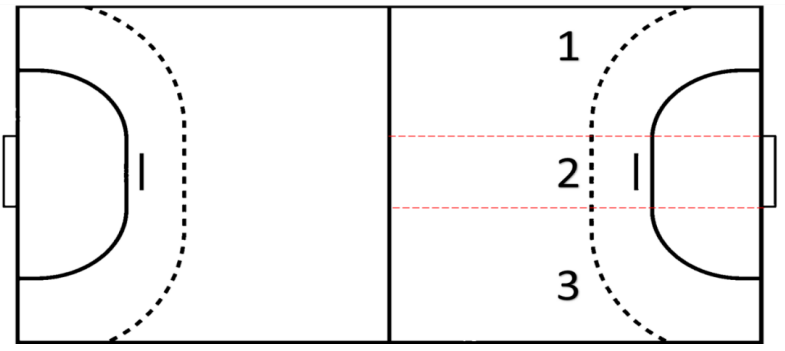

Figura 1. División de las zonas del terreno de juego

El jugador que inicia el desequilibrio es el que realiza la primera acción de ataque organizado y provoca un desajuste en la defensa. Se identifica por la posición del juego (central, lateral izquierdo, lateral derecho, extremo izquierdo, extremo derecho y pivote), y la categoría D2 es para cuando el desequilibrio lo ejecuten dos o más jugadores. Este criterio está determinado por la posición del jugador al ataque, aun cuando el desequilibrio se haya realizado en otra posición por un cruce, una circulación o por desdoblamientos.

La situación que produce el desequilibrio, presenta tres categorías; la situación de igualdad numérica se puede presentar en $1 \times 1,2 \times 2$ o $3 \times 3$, la inferioridad numérica se utiliza cuando en alguna de las tres zonas definidas, se producen situaciones de $1 \times 2,2 \times 3$ o $3 \times 4$, y la superioridad numérica respecto a la defensa se manifiesta en los casos de $2 \times 1,3 \times 1$ o $3 \times 2$.

La acción técnico-táctica individual es la acción motriz que utiliza el jugador que produce el desequilibrio para desencadenar la jugada observada. El criterio medio táctico colectivo es la acción colectiva que emplea el equipo observado para conseguir un gol o un éxito relativo, la categoría NREAL se utiliza cuando la acción iniciada es detenida por un oficial del partido, o cuando ocurrido el desequilibrio la acción finaliza sin la ejecución de ningún medio táctico colectivo. La categoría SIMPL se emplea para señalar que se ha realizado una de las siguientes acciones: el pase y va, cruce, bloqueo, pantalla, cortina y penetración sucesiva; excepto las circulaciones y los desdoblamientos. La categoría TRANS se emplea para la ocurrencia de circulación y desdoblamientos, y la categoría COMBC se utiliza en la ocurrencia de dos o más medios tácticos, exceptuando las circulaciones o desdoblamientos.

La ejecución de la decisión es el criterio que permite valorar el comportamiento de la acción tanto técnica como táctica del equipo observado. La categoría ATAATE se tendrá en cuenta cuando se ha realizado de manera correcta la ejecución técnica y táctica; ATEETA se identificará cuando el equipo ha empleado los recursos técnicos de manera adecuada, pero se ha errado la ejecución táctica. ATAETE se utiliza cuando el equipo observado ha realizado una correcta definición táctica, pero ha ejecutado la técnica de manera deficiente. ETEETA se determinará cuando se ha ejecutado con dificultad la técnica y la táctica, y la categoría DESNOB se refleja en las acciones donde haya inobservancia de decisión en la acción; cuando la acción del juego es detenida por la mesa, o cuando se decreta Golpe Franco iniciando la acción sin participación de otros jugadores del equipo observado. El criterio acción defensiva se refiere a la acción motriz que realizan uno o más jugadores defensores para evitar que el equipo contrario consiga una ejecución eficaz. En las propuestas se encuentra el Acoso, que será identificado cuando se visualice la conducta del jugador por interferir el lanzamiento, el pase o el avance del jugador en acción. En la categoría robo/interceptación se tendrán en cuenta tanto para el pase, como del drible, y para definir la ocurrencia del blocaje (BLOC) los jugadores deben realizar contacto con el balón.

Las zonas de finalización son las que se han determinado como zonas iniciales, que se codifican como ZF1, ZF2 y ZF3. En el criterio resultado final no se propuso el gol como categoría, sino la situación o condiciones con que se realizan los lanzamientos a la portería. El lanzamiento forzado (LZMFOR) se considera cuando se ha realizado un lanzamiento a portería sin tener clara posibilidad de lanzamiento. El lanzamiento ventajoso (LZMVTJ) se utiliza cuando finaliza la acción con la ejecución de un lanzamiento a puerta con clara posibilidad de ejecución. La categoría (SANCON) se utiliza para registrar las 


\section{Quiñones, Morillo-Baro, Reigal, Morales-Sánchez, Vázquez-Diz, Hernández-Mendo.}

sanciones recibidas por los jugadores que defienden las acciones del equipo observado; esa categoría prevalece por encima de otra que ocurra en la misma acción. Cada criterio y categoría están definidos por un núcleo categorial y su nivel de plasticidad (Anguera, 1990). Como ejemplo, en la tabla 2 se muestra uno de ellos.

Tabla 2. Definición de una categoría: núcleo categorial y nivel de plasticidad

SIMPL: Medio táctico simple

Núcleo categorial: Se utiliza cuando se emplea un solo medio táctico.

Nivel de plasticidad: Se marcará cuando se ejecuta uno de los siguientes medios tácticos colectivos: Pase y va, Cruces, Bloqueos, Pantallas, Cortinas, Penetración sucesiva; excepto las circulaciones y los desdoblamientos.

\section{Procedimiento}

La unidad de análisis ha sido la situación de ataque posicional en el balonmano, registrándose todas las acciones de ataque, excepto los contraataques. La unidad de análisis se inicia cuando el equipo observado sobrepasa con el balón la línea central y al poner el balón en juego en el lugar que ha finalizado la acción anterior; y finaliza, cuando termina cada acción de ataque con alguno de los resultados finales descritos. Una vez realizada la recogida de datos, el observador debe tener la garantía necesaria sobre su calidad (Anguera, 2000).

Este estudio ha realizado un análisis de calidad del dato de carácter cuantitativo estimando los coeficientes de correlación de Pearson, Spearman y Tau-B de Kendall, además para el concepto de asociación se estimó el índice Kappa de Cohen.

El proceso se realizó utilizando el programa informático HOISAN (Hernández-Mendo et al., 2012), donde el observador 1 registró los datos de los partidos Ángel Ximénez AVIA Puente Genil - BM. Benidorm y de Rincón Fertilidad Málaga-Balonmano Castellón. Pasados quince días volvió a registrar los mismos partidos y se obtuvieron los resultados de la concordancia intraobservador. Posteriormente se establecieron los acuerdos con el observador 2 realizando un entrenamiento hasta que se consideró que tenía buen control de la herramienta para registrar los datos de ambos partidos, obteniendo de esta forma los resultados de la concordancia interobservador.
Una vez superado este paso de la investigación se realizó un Análisis de Generalizabilidad a través del software informático SAGT (Hernández-Mendo, Ramos-Pérez y Pastrana, 2012). La Teoría de Generalizabilidad (Cardinet, Tourneur y Allal, 1976, 1981; Cronbach, Gleser, Nanda y Rajaratnam, 1972) unifica las definiciones de fiabilidad, validez y precisión (Blanco-Villaseñor, Castellano, HernándezMendo, Sánchez-López y Usabiaga, 2014) y está compuesta básicamente de cuatro fases: (1) Definición de las facetas de estudio; (2) Análisis de varianza de las puntuaciones obtenidas sobre las facetas de estudio; (3) Cálculo de los componentes de error; (4) Optimización de los coeficientes de Generalizabilidad (Blanco-Villaseñor, Castellano, Hernández-Mendo, Sánchez-López y Usabiaga, 2014).

\section{RESULTADOS}

A continuación, se exponen los resultados obtenidos durante la realización de este estudio en los diferentes procesos desarrollados.

\section{Concordancias}

El análisis de la Calidad del Dato se realizó desde un punto de vista cuantitativo obteniendo los coeficientes de correlación Pearson, Spearman y Tau b de Kendall, y el índice de concordancia Kappa de Cohen para ambos sexos (Tabla 3 ).

Tabla 3. Resultados de los coeficientes de correlación

Pearson, Spearman y Tau b de Kendall, y el índice de concordancia Kappa de Cohen de los partido masculino y femenino

\begin{tabular}{lcccc}
\hline \multicolumn{5}{c}{ Coeficientes de Correlación } \\
\hline Coeficiente & \multicolumn{2}{c}{ Concordancia Intra } & \multicolumn{2}{c}{ Concordancia Inter } \\
\hline & \multicolumn{1}{c}{ Masculino } & Femenino & Masculino & Femenino \\
\cline { 2 - 5 } Pearson & .99 & .97 & .98 & .98 \\
Spearman & .99 & .97 & .96 & .95 \\
Tau b de & .97 & .95 & .89 & .87 \\
Kendall & .97 & & & \\
Kappa de & .94 & .91 & .82 & .81 \\
Cohen & & & &
\end{tabular}

Los valores que se muestran en la tabla se consideran resultados adecuados para obtener registros fiables; tanto para la categoría femenina como para la masculina. 


\section{El ataque posicional en balonmano: validación de un sistema de observación}

\section{Análisis de invarianza}

La invarianza de medida se define con relación a un grupo o forma de un test, de tal modo que el significado formal y sustantivo de la medición es independiente respecto a ellos. Formalmente, la distribución de puntuaciones observadas depende sólo del espacio latente multidimensional (Mellenbergh, 1989; Meredith, 1993) del que aquellas son indicadores. Para la estimación de la invarianza se ha considerado adecuado realizar un contraste de hipótesis de las correlaciones asumiendo que Ho: $\rho_{1}-\rho_{2}=0$ y H1: $\rho_{1}-\rho_{2} \neq 0$. Se ha utilizado como estadístico de contraste la siguiente fórmula de $\mathrm{Z}$ :

$\mathrm{Z}=\frac{(1 / 2) \ln \left[\left(1+\mathrm{r}_{1}\right) /\left(1-\mathrm{r}_{1}\right)\right]-(1 / 2) \ln \left[\left(1+\mathrm{r}_{2}\right) /\left(1-\mathrm{r}_{2}\right)\right]}{\sqrt{1 /\left(\mathrm{n}_{1}-3\right)+1 /\left(\mathrm{n}_{2}-3\right)}}$

donde:

$r_{l}=$ correlación entre satisfacción y confianza

calculada en el grupo de temporales

$r_{2}=$ correlación entre satisfacción y confianza

calculada en el grupo de fijos

$n_{1}=$ tamaño de la muestra de temporales

$n_{2}=$ tamaño de la muestra de fijos

$\ln =$ logaritmo neperiano

Los resultados encontrados figuran en la Tabla 4.

Tabla 4. Resultados del contraste de hipótesis en muestras independientes y muestras relacionadas de los partido masculino y femenino.

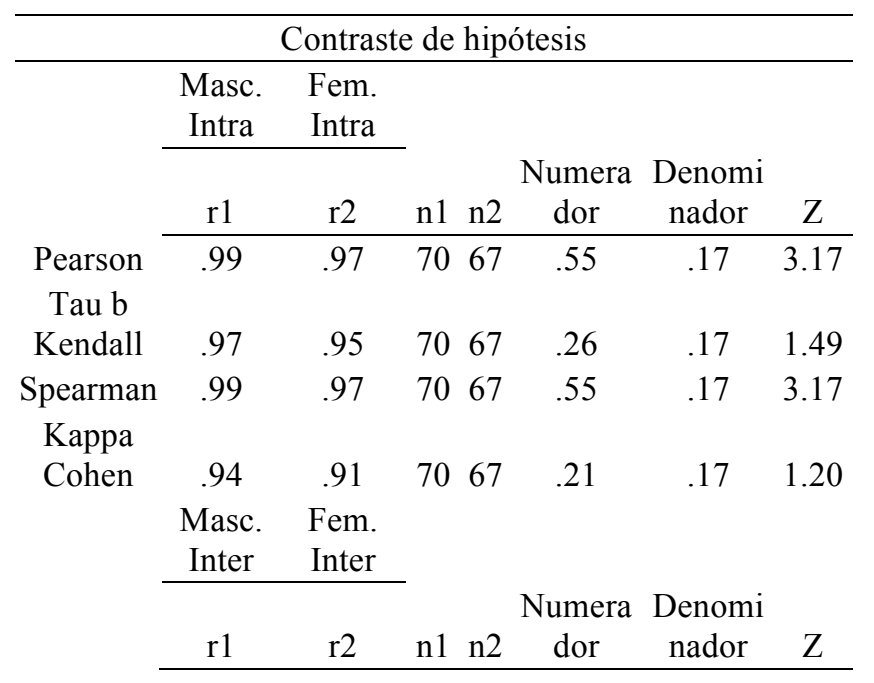

\begin{tabular}{cccccccc} 
Pearson & .98 & .98 & 70 & 67 & 0 & .17 & 0 \\
Tau b & & & & & & & \\
Kendall & .89 & .87 & 70 & 67 & .08 & .17 & .50 \\
Spearman & .96 & .95 & 70 & 67 & .11 & .17 & .65 \\
Kappa & & & & & & & \\
Cohen & .82 & .81 & 70 & 67 & .02 & .17 & .17 \\
\hline
\end{tabular}

Análisis de Generalizabilidad

Para el análisis de Generalizabilidad (Cardinet, Tourneur y Allal, 1976, 1981; Cronbach, Gleser, Nanda y Rajaratnam, 1972) se ha seguido el trabajo de Blanco-Villaseñor, Castellano, Hernández-Mendo, Sánchez-López y Usabiaga (2014). Al determinar la fiabilidad entre los observadores (concordancia interobservador) se ha empleado un diseño de dos facetas (categorías y observadores $=\mathrm{C} / \mathrm{O}$ ). $\mathrm{El}$ informe obtenido mediante el programa SAGT muestra un porcentaje de varianza asociado a la faceta Categorías de $98.03 \%$, siendo 0 para la faceta Observadores y de $1.96 \%$ en la interacción de las facetas (Categorías/Observadores). El coeficiente de generalizabilidad relativo (fiabilidad) es 0.99 y el coeficiente de generalizabilidad absoluto (generalizabilidad) es 0.99 .

Para la determinación de la fiabilidad intraobservador se ha tomado el mismo diseño. Los resultados indican que la varianza queda asociada a la faceta Categorías con $99.91 \%$, siendo 0 para la faceta Observadores y de $0.08 \%$ para la interacción de las facetas (Categorías/Observadores). El coeficiente de generalizabilidad relativo es 1.00 y el coeficiente de generalizabilidad absoluto es 1.00 .

Para estimar la homogeneidad de las categorías se ha utilizado el diseño basado en dos facetas (Observadores y Categorías $=\mathrm{O} / \mathrm{C}$ ), intentando comprobar en qué grado las categorías propuestas diferencian las acciones de ataque posicional. Los coeficientes de generalización para esta estructura de diseño son nulos (0.00); esto indica que la homogeneidad de las categorías resultan altamente significativas en el sentido de diferenciadoras.

Para la estimación del número mínimo de sesiones, necesarias para generalizar con precisión, se ha tomado un diseño de tres facetas (Observadores, Partidos y Categorías). La estimación de los componentes de varianza se ha llevado a cabo de forma aleatoria infinita. El análisis muestra que una alta variabilidad queda asociada a la faceta Categorías (69.87\%), siendo nula para la faceta Partidos (0.00), 


\section{Quiñones, Morillo-Baro, Reigal, Morales-Sánchez, Vázquez-Diz, Hernández-Mendo.}

quedando el resto de la variabilidad para la faceta de interacción Observadores/Categorías/Partidos en (0.00). Considerando los índices de generalizabilidad según esta estructura de diseño (Tabla 5), se puede determinar que con la observación de 8 partidos se obtendrían excelentes resultados con un coeficiente de generalizabilidad relativo de 0.95 y un coeficiente absoluto de 0.95 .

Tabla 5. Estudio de decisión del análisis de Generalizabilidad para estimación del número de partidos

\begin{tabular}{|c|c|c|c|c|c|c|c|c|}
\hline $\begin{array}{c}\text { Nombre } \\
\text { de los } \\
\text { valores }\end{array}$ & $\begin{array}{c}\text { Resu } \\
\text { men }\end{array}$ & $\begin{array}{c}\text { Resu } \\
\text { men } \\
2\end{array}$ & $\begin{array}{c}\text { Resu } \\
\text { men } \\
3\end{array}$ & $\begin{array}{c}\text { Resu } \\
\text { men } \\
4\end{array}$ & $\begin{array}{c}\text { Resu } \\
\text { men } \\
5\end{array}$ & $\begin{array}{c}\text { Resu } \\
\text { men } \\
6\end{array}$ & $\begin{array}{c}\text { Resu } \\
\text { men } \\
7\end{array}$ & $\begin{array}{c}\text { Resu } \\
\text { men } \\
8\end{array}$ \\
\hline 0 & $\begin{array}{l}(2 ; \\
\text { INF) }\end{array}$ & $\begin{array}{c}(2 ; \\
\text { INF) }\end{array}$ & $\begin{array}{c}(2 ; \\
\text { INF) }\end{array}$ & $\begin{array}{c}(2 ; \\
\text { INF) }\end{array}$ & $\begin{array}{c}(2 ; \\
\text { INF) }\end{array}$ & $\begin{array}{c}(2 ; \\
\text { INF) }\end{array}$ & $\begin{array}{c}(2 ; \\
\text { INF) }\end{array}$ & $\begin{array}{c}(2 ; \\
\text { INF) }\end{array}$ \\
\hline $\mathrm{C}$ & $\begin{array}{l}(62 ; \mathrm{I} \\
\mathrm{NF})\end{array}$ & $\begin{array}{l}(62 ; \mathrm{I} \\
\mathrm{NF})\end{array}$ & $\begin{array}{l}(62 ; \mathrm{I} \\
\mathrm{NF})\end{array}$ & $\begin{array}{l}(62 ; \mathrm{I} \\
\mathrm{NF})\end{array}$ & $\begin{array}{l}(62 ; \mathrm{I} \\
\mathrm{NF})\end{array}$ & $\begin{array}{l}(62 ; \mathrm{I} \\
\mathrm{NF})\end{array}$ & $\begin{array}{l}(62 ; \mathrm{I} \\
\mathrm{NF})\end{array}$ & $\begin{array}{l}(62 ; \mathrm{I} \\
\mathrm{NF})\end{array}$ \\
\hline $\mathbf{p}$ & $\begin{array}{c}1 \\
\text { (INF) }\end{array}$ & $\begin{array}{c}2(\mathrm{IN} \\
\mathrm{F})\end{array}$ & $\begin{array}{c}3 \\
\text { (INF) }\end{array}$ & $\begin{array}{c}4 \\
\text { (INF) }\end{array}$ & $\begin{array}{c}5 \\
\text { (INF) }\end{array}$ & $\begin{array}{c}6 \\
\text { (INF) }\end{array}$ & $\begin{array}{c}7 \\
\text { (INF) }\end{array}$ & $\begin{array}{c}8 \\
\text { (INF) }\end{array}$ \\
\hline $\begin{array}{c}\text { Total } \\
\text { observaci } \\
\text { ones }\end{array}$ & 62 & 124 & 186 & 248 & 310 & 372 & 434 & 496 \\
\hline $\begin{array}{c}\text { Coeficien } \\
\text { te } G \\
\text { relativo }\end{array}$ & 0.69 & 0.82 & 0.87 & 0.90 & 0.92 & 0.93 & 0.94 & 0.95 \\
\hline $\begin{array}{c}\text { Coeficien } \\
\text { te G } \\
\text { absoluto }\end{array}$ & 0.69 & 0.82 & 0.87 & 0.90 & 0.92 & 0.93 & 0.94 & 0.95 \\
\hline
\end{tabular}

\section{DISCUSIÓN}

El objetivo de este estudio se centró en diseñar una herramienta de observación para analizar los comportamientos de los jugadores de balonmano en el ataque posicional, así como interpretar los resultados devenidos de la fiabilidad, validez y precisión de la herramienta, como indicadores de calidad de los datos recogidos a través de ella.

Los resultados muestran que la herramienta de observación diseñada recoge los criterios necesarios para analizar el comportamiento de los jugadores y jugadoras de balonmano en el ataque posicional. A pesar de la originalidad del sistema de observación, algunas categorías se asemejan a las propuestas en otros estudios realizados en balonmano (Flores y Anguera, 2018; González, 2012; Lozano, Camerino e Hileno, 2016; Morgado, 2012; Morillo y HernándezMendo, 2015; Prudente, Sousa, Sequeira, LópezLópez y Hernández-Mendo, 2017; Salas y Hernández-
Mendo, 2016; Sousa, Prudente, Sequeira, LópezLópez y Hernández-Mendo, 2014).

Concretamente, en el diseño de la herramienta se decidió agrupar el criterio Medios Tácticos Colectivos en cuatro categorías, decisión tomada por la complejidad de registrar el elevado número comportamientos a observar y a la velocidad con que ocurren las acciones en el juego de competición en balonmano. En la investigación realizada por Lozano, Camerino e Hileno (2016) se presentan los medios tácticos en dos categorías: medios tácticos básicos y medios tácticos complejos. En este estudio, con la intención de alcanzar un mayor grado de exhaustividad, las conductas se han agrupado en cuatro categorías: medio táctico no realizado (NREAL), medios tácticos simples (SIMPL), las transformaciones (TRANS) y medios tácticos combinados (COMBC).

El equilibrio numérico permite analizar la composición numérica entre atacantes y defensores en una situación de juego concreta. En este estudio se analiza ante la primera acción del ataque a diferencia de otros estudios que también contemplan un criterio similar tanto en balonmano como en balonmano playa (Lozano y Camerino, 2012; Morillo y HernándezMendo, 2015; Salas y Hernández-Mendo, 2016; y Lozano, Camerino e Hileno, 2016). Se consideró proponer tres categorías con las posibilidades que se presentan al interactuar atacantes y defensores: situación de igualdad numérica $(1 \times 1)$, situaciones de inferioridad numérica $(1 \times 2)$ y las situaciones de superioridad numérica $(2 \times 1)$; categorías similares a las propuestas por González, Botejara, Martínez y Chirosa (2016).

Se determinó incorporar un criterio de significativa importancia; el criterio ejecución de la decisión, que permite desde la observación analizar la calidad con que se producen las acciones técnico-tácticas durante el ataque posicional. Se determinaron cinco categorías que describen las posibles opciones: acierto táctico y acierto técnico para todas las acciones que se realicen de manera acertada tanto en la ejecución técnica como en la decisión táctica. Las categorías acierto técnico y error táctico, y acierto táctico y error técnico se propusieron para las acciones que se observaran ante estas condiciones. La categoría error técnico y error táctico fue incorporado para las acciones de juego que se observaran errores en la técnica y en la decisión táctica. En este criterio, el gol no condiciona la determinación de las categorías, la condición está 


\section{El ataque posicional en balonmano: validación de un sistema de observación}

mediada por la calidad con que se ejecute la acción en su conjunto. Como última categoría, se decidió incluir decisión técnico y táctica no observada, para registrar las acciones de ataque que eran interrumpidas al iniciar el ataque y la acción no lograba tener continuidad.

Un criterio que ha sido incorporado con frecuencia en las herramientas observacionales de balonmano sobre los comportamientos de los jugadores en su actuar ofensivo ha sido el resultado de final, y como conducta referente el gol. En este estudio, no se propone el gol como categoría de este criterio, sino que fue oportuno analizar las condiciones en que se ejecuta el lanzamiento: lanzamiento ventajoso (LZMVTJ) y lanzamiento forzado (LZMFOR). Este análisis se interpretó de forma similar en la investigación de Lozano, Camerino y Hieleno (2016) donde se determinó si hubo una clara ocasión de gol o si hubo una mala elección del lanzamiento.

Durante el desarrollo de este estudio se presentaron las dificultades propias de los deportes de equipo: la gran cantidad de información a registrar y la velocidad en que ocurren las acciones en el juego (Morillo Baro y Hernández-Mendo, 2015); resultado de las interacciones colectivas de los jugadores de balonmano en su contexto natural, la competición. En consecuencia, estas dificultades se manifestaron al registrar las zonas iniciales y finales de las acciones. A pesar de ello, los resultados de los análisis muestran índices adecuados de los coeficientes de correlación y de los valores obtenidos en el análisis de generalizabilidad, poniendo de relieve que la herramienta de observación diseñada para analizar los comportamientos en el ataque posicional en balonmano permite registrar de forma fiable, válida y precisa. Además se constata en el análisis obtenido del cálculo de invarianza (tabla 4) que no existen diferencias por razón de género en la utilización de la herramienta.

\section{APLICACIONES PRÁCTICAS}

$\mathrm{Al}$ cumplir con los objetivos propuestos en el estudio, las posibles aplicaciones prácticas que aparecen están vinculadas al estudio de los comportamientos tácticos de los jugadores en diferentes categorías y niveles de competición. Al igual que en estudios recientes, el análisis secuencial y de coordenadas polares mediante la observación sistemática permite contemplar el parámetro orden en la ejecución, valor destacado de los estudios dinámicos y con enorme repercusión para el conocimiento del juego.

\section{REFERENCIAS}

1. Anguera, M. T. (1979). Observación de la conducta espacial. Comunicación presentada al VI Congreso Nacional de Psicología, Pamplona, España.

2. Anguera, M. T. (2000). Del registro narrativo al análisis cuantitativo: Radiografía de la realidad perceptible. En Ciencia i cultura en el segle XXI. Estudis en homenatge a Josep Casajuana (pp. 4171). Barcelona: Reial Academia de Doctors.

3. Anguera, M. T., Blanco, A., Hernández-Mendo, A. y Losada, J. L. (2011). Diseños observacionales: ajuste y aplicación en psicología del deporte. Cuadernos de Psicología del Deporte, 11 (2), 63-76.

4. Anguera, M. T. y Hernández-Mendo, A. (2013). La metodología observacional en el ámbito del deporte. E-balonmano.com: Revista de Ciencias del Deporte 9(3), 135-160. Recuperado de www.e-

balonmano.com/ojs/index.php/revista/about

5. Anguera, M. T. y Hernández-Mendo, A. (2014). Metodología observacional y psicología del deporte: Estado de la cuestión. Revista de Psicología del Deporte, 23(1), 103-109.

6. Anguera, M. T. y Hernández-Mendo, A. (2015). Técnicas de análisis en estudios observacionales en ciencias del deporte. Cuadernos de Psicología del Deporte, 15(1), 13-30. https://doi.org/10.4321/S157884232015000100002

7. Blanco-Villaseñor, A., Castellano, J., HernándezMendo, A., Sánchez-López, C. R. y Usabiaga, O. (2014). Aplicación de la TG en el deporte para el estudio de la fiabilidad, validez y estimación de la muestra. Revista de Psicología del Deporte, 23(1), 131-137.

8. Cardinet, J. Tourneur, Y. \& Allal, L. (1976). The simmetry of generalizability theory: Applications 


\section{Quiñones, Morillo-Baro, Reigal, Morales-Sánchez, Vázquez-Diz, Hernández-Mendo.}

to educational measurement. Journal of Educational Measurement, 13(2), 119-135. https://doi.org/10.1111/j.17453984.1976.tb00003.x

9. Cardinet, J. Tourneur, Y. \& Allal, L. (1981). Extension of generalizability theory and its applications in educational measurement. Journal of Educational Measurement, 18(4), 183-204. https://doi.org/10.1111/j.1745$\underline{\text { 3984.1981.tb00852.x }}$

10. Casal, C. A., Maneiro, R., Ardá, T., Losada, J. L. y Rial, A. (2014). Effectiveness of Indirect Free Kicks in Elite Soccer. International Journal of Performance Analysis in Sport, 14, 744-750. https://doi.org/10.1080/24748668.2014.1186875 $\underline{5}$

11. Castellano, J. (2000). Observación y análisis de la acción de juego en fútbol. Lecturas: EF y Deportes. Revista Digital, 5(22). Recuperado de http://www.efdeportes.com/efd22b/julentd.htm

12. Cronbach, L.J., Gleser, G.C., Nanda, H. \& Rajaratnam, N. (1972). The dependability of behavioral measurements: theory of generalizability for scores and profiles. New York: John Wiley and Sons.

13. Flores, J. y Anguera, M. T. (2018). Patrón de juego en balonmano según el jugador que ocupa la posición de central. Apunts: Educación Física y Deportes, 134(4),

110-123.

https://doi.org/10.5672/apunts.2014-

0983.cat.(2018/4).134.08

14. González, A. (2012). Análisis de la eficacia del contraataque en balonmano como elemento de rendimiento deportivo. (Tesis doctoral). Universidad de León. León.

15. González Ramírez, A. (2016). Fiabilidad de un instrumento de observación para analizar el rendimiento en Handball. Revista Ciencias Psicológicas, 10(2), ISSN: 1688-4094 / 16884221

16. González Ramírez, A., Botejara Lemos, J. L., Martínez Martín, I. y Chirosa Ríos, L. J. (2016).
Eficacia del ataque y del lanzamiento de los cuatro primeros clasificados en balonmano masculino de los Juegos ODESUR 2014. Educación Física y Ciencia, 18(1), e004. Recuperado de http://www.efyc.fahce.unlp.edu.ar/article/view/E $\underline{\text { FyCv18n01a04/ }}$

17. Hernández-Mendo, A. (1996). Observación y análisis de patrones de juego en deportes sociomotores. (Tesis Doctoral sin publicar). Universidad de Santiago de Compostela, Santiago de Compostela, España.

18. Hernández-Mendo, A., López-López, J., Castellano, J., Morales-Sánchez, V. y Pastrana, J. L. (2012). Hoisan 1.2: Programa informático para uso en metodología observacional. Cuadernos de Psicología del Deporte, 12 (1), 55-78. https://doi.org/10.4321/S1578$\underline{84232012000100006}$

19. Hernández-Mendo, A., Ramos-Pérez, F. y Pastrana, J. L. (2012). SAGT: Programa informático para análisis de Teoría de la Generalizabilidad. SAFE CREATIVE Código: 1204191501059.

20. Jiménez-Salas, J. y Hernández-Mendo, A. (2016). Análisis de la calidad del dato y generalizabilidad de un sistema de observación del contraataque en el balonmano de élite. E-balonmano.com: Revista de Ciencias del Deporte 12(1), 31-34. http://www.ebalonmano.com/ojs/index.php/revista/index

21. Lozano, D. y Camerino, O. (2012). Eficacia de los sistemas ofensivos en balonmano. Apunts: Educación física y deportes (108), 70-81. https://doi.org/10.5672/apunts.20140983.es.(2012/2).108.08

22. Lozano, D. (2014). Análisis del comportamiento táctico ofensivo en alto rendimiento en balonmano. (Tesis doctoral). Universidad de Lleida. Recuperado de http://hdl.handle.net/10803/283756

23. Lozano, D., Camerino, O. y Hileno, R. (2016). Análisis del comportamiento táctico ofensivo en momentos críticos de juego en el alto rendimiento 


\section{El ataque posicional en balonmano: validación de un sistema de observación}

en balonmano: un estudio Mixed Methods. Cuadernos de Psicología del Deporte, 16(1), 151160. ISSN 1989-5879

24. Marques de Sousa, A. (2011). O comportamento da defesa da Selecção do Espanha no Torneio de Andebol en Pequim 2008. Análise seqüencial no método organizado de jogo de andebol em situação de 6x6. (Tesis doctoral). INEFC Lleida.

25. Mellenbergh, G. J. (1989). Item bias and item response theory. International Journal of Educational Research, 13, 127-143. https://doi.org/10.1016/0883-0355(89)90002-5

26. Meredith, W. (1993). Measurement invariance, factor analysis and factorial invariance. Psychometrika, 58, 525-543. https://doi.org/10.1007/BF02294825

27. Montoya, M., Moras, G. y Anguera, M. T. (2013). Análisis de las finalizaciones de los jugadores extremo en balonmano. Apunts: Educación Física y Deportes, 113, 52-59. https://doi.org/10.5672/apunts.20140983.es.(2013/3).113.05

28. Moreno, E. y Gómez, M. A. (2017). Validación herramienta observacional para el análisis de rachas de lanzamiento en baloncesto. Revista de Psicología del Deporte / Journal of Sport Psychology. 26(1), 87-93.

29. Morgado, A. P. (2012). Análisis de los factores de eficacia de las acciones de prefinalización y finalización en ataque organizado en balonmano de alto nivel. (Tesis doctoral). Universidad de Castilla-La Mancha. Toledo, España.

30. Morillo Baro, J.P. y Hernández- Mendo, A. (2015). Análisis de la calidad del dato de un instrumento para la observación del ataque en balonmano playa. Revista Iberoamericana de Psicología del Ejercicio y el Deporte. 10(1), 1522.

31. Muñoz, V. y Serna, J. (2015). Diseño, fiabilidad y validez del instrumento de observación SOCCB para el análisis de las finalizaciones en baloncesto. Cuadernos de Psicología del Deporte, 15(3), 169-
174. $\underline{84232015000300017}$

32. Ortega-Toro, E., García-Angulo, A., GiménezEgido, J.M., García-Angulo, F.J. \& Palao, J.M. (2019). Design, Validation, and Reliability of an Observation Instrument for Technical and Tactical Actions of the Offense Phase in Soccer. Front. Psychol. 10:22. https://doi: 10.3389/fpsyg.2019.00022

33. Pajares, I. C., Echeverría, C. F., Silva, J. G., Suárez, M. C. y Arroyo, M. P. M. (2017). Estudio predictivo de la eficacia de la recepción en voleibol juvenil masculino. Retos: Nuevas tendencias en Educación Física, Deporte y Recreación, (32), 214-218.

34. Pic, M. (2017). The observation of gender differences in handball. E-Balonmano.com: Revista de Ciencias del Deporte, 13 (3), 191-198. ISSN $1885-7019$

35. Prieto, J., Gómez, M. A. y Sampaio, J. (2015a). From a Static to a Dynamic Perspective in Handball Match Analysis: a Systematic Review. The Open Sports Sciences Journal, 8(1), 25-34. https://doi.org/10.2174/1875399X01508010025

36. Prieto, J., Gómez, M. A. y Sampaio, J. (2015b). Revisión bibliométrica de la producción científica en balonmano. Cuadernos de Psicología del Deporte, 15(3), 145-154. https://doi.org/10.4321/S1578$\underline{84232015000300014}$

37. Prudente, J., Sousa, D., Sequeira, P., LópezLópez, J. A. y Hernández-Mendo, A. (2017). Analyzing the influence of playing time and partial score on the tactical behavior in the duel 2 vs 2 in the offensive process in handball, using the polar coordinates technique. Anales de psicología, 33(3),

515-529. https://doi.org/10.6018/analesps.33.3.271071

38. Salas, J. y Hernández-Mendo, A. (2016). Análisis de la calidad del dato y generalizabilidad de un sistema de observación del contraataque en el balonmano de élite. E-Balonmano.com: Revista 


\section{Quiñones, Morillo-Baro, Reigal, Morales-Sánchez, Vázquez-Diz, Hernández-Mendo.}

de Ciencias del Deporte, [S.1.], 12(1), 31-44. ISSN $1885-7019$

39. Sá, P., Rui, A., Saavedra, M. y Fernández, J. J. (2015). Percepción de los porteros expertos en balonmano de los factores determinantes para el éxito deportivo. Revista de Psicología del Deporte, 24(1), 21-27.

40. Santos, S., Sarmento, H., Alves, J. y Campaniço, J. (2014). Construcción de un instrumento para la observación y el análisis de las interacciones en el waterpolo. Revista de Psicología del Deporte. Vol. 23(1), 191-200.

41. Sarmento, H., Marcelino, R., Anguera, M. T., Campaniço, J., Matos, N. \& Leitão, J. C. (2014). Match analysis in football: a systematic review. Journal of Sports Sciences, 1-13. doi.org/10.1080/02640414.2014.898852

42. Sousa, D., Prudente, J., Sequeira, P. y HernándezMendo, A. (2014). Análise da qualidade dos dados de um instrumento para observação do 2 vs 2 no andebol. Revista Iberoamericana de Psicología del Ejercicio y el Deporte, 9(1), 173-190.

43. Tassi, J. M., Rivera, S. y Morilla, M. (2018). El Entrenamiento Psicológico-Integrado en Fútbol a través de Tareas de Entrenamiento. Revista de Psicología Aplicada al Deporte y al Ejercicio Físico, 3, e5, 1-15 https://doi.org/10.5093/rpadef2018a4

44. Teles, N. y Volossovitch, A. (2015). Influência das variáveis contextuais no desempenho das equipes nos últimos 10 minutos do jogo de handebol. Revista Brasileira de Educação Física e Esporte, 29(2), 177-187. https://doi.org/10.1590/180755092015000200177

45. Tulha Duarte Ferreira, A.L., Braga Santos Graça, A. e Dias Estriga, M. L. (2018). Desenvolvimento e validação de um sistema de avaliação técnicotático individual no andebol: the handball tactical performance evaluation (htpe). Ebalonmano.com: Revista de Ciencias del Deporte, 14 (3), 131-140. http://www.ebalonmano.com/ojs/index.php/revista/index
46. Vázquez-Diz, J. A., Morillo-Baro, J. P., Reigal, R. E., Morales-Sánchez, V. y Hernández-Mendo, A. (2019a). Diseño y validación de una herramienta de observación para porteros en balonmano playa. Cuadernos de Psicología del Deporte, 19(2), 135146.

47. Vázquez-Diz, J. A., Morillo-Baro, J. P., Reigal, R. E., Morales-Sánchez, V. \& Hernández-Mendo, A. (2019b). Mixed Methods in Decision-Making Through Polar Coordinate Technique: Differences by Gender on Beach Handball Specialist. Front. Psychol. 10:1627. doi: 10.3389/fpsyg.2019.01627

48. Vázquez-Diz, J. A., Morillo-Baro, J. P., Reigal, R. E., Morales-Sánchez, V. \& Hernández-Mendo, A. (2019c). Contextual Factors and Decision-Making in the Behavior of Finalization in the Positional Attack in Beach Handball: Differences by Gender Through Polar Coordinates Analysis. Front. Psychol. 10:1386. doi: 10.3389/fpsyg.2019.01386

49. Villarejo, D., Ortega, E., Gómez, M. A. \& Palao, J. M. (2014). Design, validation, and reliability of an observational instrument for ball possessions in rugby union. International Journal of Performance Analysis in Sport, 14 (3), 955967. https://doi.org/10.1080/24748668.2014.1186877 $\underline{1}$

50. Vukosavljević, J., Kocić, M., Beric, D. \& Stojić, M. (2015). The situation success in the handball: Review. Facta Universitatis, Series: Physical Education and Sport, 13(1), 97-105. 